\title{
Faune du sol et microflore. II. Saprophagie, prédation et médiation chimique
}

\author{
M Pussard \\ INRA, station de recherches sur la faune du sol, 17, rue Sully, 21034 Dijon Cedex, France
}

(Reçu le 16 mai 1990; accepté le 13 mars 1991)

\begin{abstract}
Résumé - Puisque la fourniture de ressources trophiques supplémentaires ne peut expliquer totalement la stimulation de la microflore par les animaux, d'autres mécanismes doivent intervenir. En premier lieu, les animaux pourraient, par prédation, agir sur les niveau des populations microbiennes dont ils modifieraient ainsi l'activité. La bactériophagie, évidente chez beaucoup de protozoaires, ne représente, chez la plupart des invertébrés saprophages, qu'un mode d'alimentation mal établi, non susceptible de modifier le niveau des populations bactériennes et d'expliquer l'effet de stimulation. À l'inverse, la mycophagie est pratiquée par de nombreux invertébrés mais elle semble provoquer plutôt une diminution de l'activité fongique. L'étude expérimentale des relations faune-bactéries est plus simple dans le cas des protozoaires. Ainsi, un travail récent a permis de montrer que ces protistes stimulent l'activité bactérienne, non par la valeur nutritive de leurs excrétions ou par une modification de la densité de population bactérienne, mais par l'émission de substances stimulantes. Ce résultat important pourrait avoir des applications en lutte microbiologique et en biotechnologie. Par extension, un nouveau mécanisme peut être envisagé dans le cas de la faune dans son ensemble : les invertébrés comme les protozoaires émettraient des substances stimulantes actives sur le métabolisme bactérien. Mais pour le prouver, il faut d'abord disposer de techniques fiables d'axénisation des invertébrés.
\end{abstract}

faune du sol / relation animal-microflore / stimulation microbienne / régime alimentaire / médiateur chimique

Summary - Soil animals and microflora. II. Saprophagy, predation and chemical mediation. Since food relationships do not explain all the animal-mediated increase in microflora activity, we must look for other mechanisms (Pussard, 1991). Microbial-feeding animals appear to modify the density and therefore the activity of the prey population. Bacteriophagy is obvious in many protozoa but in saprophagic invertebrates it is less clear and probably cannot modify bacterial population density and activity. Inversely mycophagy is very frequent in invertebrates but tends to bring about a decrease in fungal activity. A study of fauna-bacteria relationships is easier with protozoa. A recent study, showed that amoebae do not increase bacterial activity by the nutritive value of their excreta or by population control, but by the release of stimulating substances. This result has led us to propose a new mechanism to explain faunal impact on bacterial microflora. Invertebrates such as amoebae may release stimulating substances. In order to verify this hypothesis, reliable techniques must be available for the production of axenic invertebrates.

soil fauna / animal-microflora relationships / microbial stimulation / diet / chemical mediator

\section{INTRODUCTION}

Dans la première partie de cet exposé (Pussard, 1991), nous avons mis l'accent sur le décalage paradoxal qui existe entre le niveau d'activité métabolique reconnu à la faune et le niveau de stimulation de la microflore observé en présence d'animaux. Cette stimulation est généralement attribuée à la fourniture par la faune de ressources trophiques supplémentaires (excrétions, sécrétions, transfert et démasquage de substrats) utilisables par la microflore. Cependant, l'examen des caractérisques générales du modèle animal nous a conduit à émettre de sérieuses réserves quant à la valeur d'une telle théorie.

Nous nous proposons donc ici de rechercher d'autres hypothèses pour expliquer le paradoxe fonctionnel. II est possible en particulier que la faune agisse par la prédation sur le niveau des populations microbiennes, en provoquant ainsi chez ces dernières une réaction métabolique dépendant de la densité de population. Ceci nous conduira à examiner l'état des connaissances re- 
latives à la bactériophagie et à la mycophagie chez les animaux et en particulier chez les espèces saprophages. Puis, pour étudier l'effet de la prédation sur l'activité de la population de proies, nous prendrons comme exemple le cas le plus simple, celui des protozoaires bactériophages. Nous serons amenés à rejeter le mécanisme dépendant de la densité et à proposer une nouvelle hypothèse, celle de l'existence de médiateurs chimiques.

\section{Importance de la consommation de biomasse microbienne par la faune}

Les espèces phytophages et les espèces carnassières mises à part, il est généralement admis que les animaux du sol peuvent avoir 3 types de régime alimentaire : la bactériophagie, la mycophagie et la saprophagie. Cette dernière traduit en fait l'ignorance de la nature de la ressource exploitée. En effet, sont généralement considérés comme saprophages au sens large les animaux du sol qui ne sont ni carnassiers, ni phytophages et qui n'ingèrent pas les propagules fongiques avec leur paroi. Le tube digestif de ces espèces est soit optiquement vide (espèces microphages, tels de nombreux nématodes), soit encombré de débris végétaux et/ou de terre (animaux saprophages au sens strict, tels les lombriciens). Appartenant à cette dernière catégorie, les espèces géophages ingèrent essentiellement de la terre et utilisent la matière organique plus ou moins étroitement associée aux éléments minéraux.

La nature exacte de la ressource trophique exploitée par les animaux saprophages qui ingèrent un matériel particulièrement complexe fait l'objet d'un débat qui a pu paraître purement académique (Swift et al, 1979). Cependant, dans le cadre de nos préoccupations, il serait utile de savoir si ces animaux sont susceptibles de consommer assez de microorganismes pour agir de façon significative sur la densité de population de ces derniers et modifier ainsi leurs activités.

La consommation de corps microbiens par les animaux pose un problème de terminologie. Le terme de "broutage" et son équivalent anglosaxon "grazing" sont souvent employés à la place du terme de prédation, sans doute sous le prétexte de l'affinité végétale reconnue jadis aux bactéries et aux champignons. En se référant précisément au broutage de l'herbe par les ruminants, nous considérons que, chez les microorganismes aussi, le broutage devrait désigner l'in- gestion d'une partie seulement de la proie, la survie de cette dernière n'étant pas systématiquement compromise. En revanche, la prédation suppose l'ingestion totale de l'individu (ou colony forming unit) avec la mort de ce dernier : ainsi bactériophagie et mycophagie relèvent bien de la prédation.

\section{Bactériophagie}

En raison de son importance dans la plupart des milieux, la biomasse bactérienne représente une considérable ressource trophique potentielle. Dans quelle mesure est-elle utilisée par la faune?

Du fait de la faible taille et des particularités structurales des bactéries, le protoplasme bactérien n'est pas accessible par simple effraction mécanique et sa digestion suppose l'intervention de substances bactéricides ou d'enzymes spécifiques capables de lyser la paroi bactérienne (type lysozyme).

La bactériophagie est infiniment mieux établie chez les protozoaires que chez les invertébrés. En effet, chez les premiers la consommation de bactéries peut être constatée directement : l'ingestion et la lyse bactérienne sont faciles à observer in vivo sous le microscope. II faudra donc examiner avec une attention particulière le rôle des protozoaires dans le contrôle des populations bactériennes et dans la stimulation de ces dernières.

À l'inverse, chez les invertébrés, la bactériophagie est beaucoup plus difficile à mettre en évidence puisque la démonstration est obligatoirement indirecte. Quatre types de preuve ont été avancés.

\section{Utilisation de l'aliment bactérien en élevage}

Ce premier type de preuve consiste à obtenir la survie, la croissance et même le développement complet de l'invertébré aux dépens du seul aliment bactérien. C'est ainsi qu'à pu être démontrée la bactériophagie chez les nématodes. Le point faible d'une telle démonstration réside dans l'absence de désinfection des animaux mis en élevage (Zobell et Feltham, 1938; Flack et Hartenstein, 1984) ou dans l'insuffisance du procédé de désinfection. Dans les 2 cas, des protozoaires risquent de contaminer l'élevage sous forme de kystes qui chez certaines espèces peuvent être très résistants aux antiseptiques utilisés (hypo- 
chlorite, dérivés mercuriques). À l'insu de l'expérimentateur, ces protozoaires se multiplieraient de façon intense aux dépens des bactéries et, à l'état de trophozoïtes, constitueraient des proies facilement digérées par les animaux (Piearce et Phillips, 1980; Rouelle, 1983). Les protozoaires serviraient alors d'intermédiaires trophiques entre bactéries et invertébrés : cette éventualité a été évoquée par les nématologistes (Stephenson, 1942; Sohlenius, 1968; Elliot et al, 1980) tandis qu'elle semble avoir échappé aux spécialistes de vers de terre (Flack et Hartenstein, 1984). Pourtant divers auteurs (Miles, 1963; Duhlinska, 1979; Rouelle, 1983) ont montré que le lombricien Eisenia fetida avait une croissance très ralentie et ne parvenait pas à l'état adulte dans un environnement microbien privé de protozaires.

\section{Utilisation de biomasse bactérienne marquée}

Un deuxième type de preuve de la bactériophagie utilise le passage d'un élément radioactif de la biomasse bactérienne dans les tissus de l'invertébré (Hargrave, 1970; Reyes et Tiedje, 1976; Harper et al, 1981a; Bignell, 1989). Malheureusement, les auteurs ne prennent aucune précaution particulière pour éviter que les protozoaires ne servent d'intermédiaires dans le tranfert du marqueur.

\section{Évolution du nombre de bactéries au cours du transit digestif}

Un troisième type de preuve, le plus ancien, consiste à comparer les densités de populations bactériennes mesurées en fin de transit digestif à celles observées soit dans la partie antérieure du tube digestif, soit dans un échantillon de milieu ambiant : I'hypothèse implicitement admise dans ce dernier cas est que l'animal ingère sans choisir. Diverses causes d'erreur interviennent dans une telle démarche : ignorance de la densité bactérienne réellement ingérée; variation de l'état de dispersion du bol alimentaire (et des bactéries) en fonction du traitement mécanique (gésier des lombriciens) ou des propriétés physicochimiques du liquide digestif; vitesse de transit variable suivant la taille des particules; multiplication des bactéries dans certains segments du tube digestif; activité lytique des actinomycètes souvent abondants dans le tube digestif des invertébrés (Parle, 1963; Kristufek et al, 1989; Chu et al, 1987; Szabo, 1989). Chez certaines espèces, cette méthode a permis d'observer effectivement une diminution de l'effectif bactérien (Wavre et
Brinkhurst, 1971; Baker et Bradnam, 1976; Lopez et Levinton, 1978; Wotton, 1980; Harper et al, 1981b; Austin et Baker, 1988); chez d'autres invertébrés tels les lombriciens (Bassalik, 1913; Cameron, 1932; Dawson, 1948; Day, 1950; Brüsewitz, 1959; Parle, 1963), c'est au contraire une forte augmentation du peuplement bactérien qui a été constatée. Dans tous les cas, ce procédé ne permet pas de conclure à la réalité de la bactériophagie.

\section{Activités bactériolytiques}

La dernière sorte de preuve consiste à mettre en évidence dans le tube digestif des invertébrés des activités enzymatiques capables de lyser la paroi bactérienne (Zobell et Feltham, 1938; McHenery et al, 1979; Childs et Bowman, 1981; Malke, 1965) ou des substances bactéricides comme la peroxydase du tube digestif de certains lombriciens (Hassett et al, 1988). Malheureusement de tels travaux ne tiennent jamais compte de l'interférence possible des protozoaires ou d'autres microorganismes lytiques associés à l'animal.

\section{En conclusion}

La bactériophagie, si elle est évidente chez les protozoaires, est rarement établie en toute rigueur chez les invertébrés; de plus, elle ne semble pas pouvoir couvrir une part importante des besoins alimentaires de ces animaux (cf $\mathrm{La}$ saprophagie).

Chez les vertébrés, en liaison avec le développement de l'axénie, les connaissances sont plus précises : ainsi chez les mammifères monogastriques, il a été établi que le tube digestif d'individus axéniques peut héberger expérimentalement n'importe quelle souche bactérienne (Ducluzeau et Raibaud, 1979), ce qui montre qu'un tel animal ne possède pas d'activité enzymatique susceptible de lyser toute bactérie en transit. Ainsi, le compartiment digestif agirait essentiellement en favorisant de façon sélective le développement des populations bactériennes tandis que les dispositifs bactériolytiques seraient affectés à la protection des autres compartiments de l'organisme. On peut supposer qu'il en est de même chez de nombreux invertébrés. Pour toutes ces raisons, la réalité de la bactériophagie ne peut être acceptée qu'au prix d'une démonstration sans faille. Seuls les proto- 
zoaires sont incontestablement impliqués dans la consommation de biomasse bactérienne.

\section{La mycophagie}

En milieu édaphique, la biomasse fongique constitue une ressource trophique importante, susceptible d'être exploitée par de nombreux organismes. Les invertébrés surmontent l'obstacle de la paroi fongique par effraction mécanique grâce à des stylets perforants (nématodes) ou des pièces buccales broyeuses (arthropodes). Chez eux la mycophagie est établie de façon beaucoup plus sûre que la bactériophagie puisque les organes fongiques en voie de digestion sont repérables dans le tube digestif après dissection. De plus, leur tube digestif est le plus souvent associé à une microflore capable d'effectuer la lyse enzymatique de la paroi fongique. La mycophagie est très répandue, en particulier chez les nématodes, les acariens et les collemboles.

Chez les protozoaires, il existe une mycophagie opportuniste qui concerne les propagules dont la forme et les dimensions permettent une phagocytose normale (spores, levures). Certaines espèces par contre sont des mycophages spécialisées : elles possèdent un comportement phagocytaire particulier qui leur permet d'utiliser le protoplasme mycélien (Old, 1977; Pussard et al, 1979; Pussard et al, 1980; Old et Darbyshire, 1980; Foissner et Didier, 1983). Toutefois, les espèces mycophages strictes sont peu nombreuses et leurs populations ne semblent pas capables de modifier de façon appréciable la densité des peuplements fongiques (Alabouvette et al, 1981).

\section{La saprophagie}

Comme nous l'avons indiqué ci-dessus, la nature des aliments assimilés par les animaux saprophages n'est pas connue avec exactitude. Un rythme d'ingestion élevé (Fenchel, 1972) permet de supposer que ces animaux n'utilisent qu'une très faible fraction du matériel ingéré. Par ailleurs, ils ingèrent plus volontiers la matière organique lorsqu'elle est colonisée par les microorganismes (Kaushik et Hynes, 1971; Wright, 1972; MacKay et Kalff, 1973; Calow, 1974; Bärlocher et Kendrick, 1975; Cooke et Luxton, 1980). Une première hypothèse consiste à supposer que les animaux se nourrissent aux dé- pens de la biomasse bactérienne. Certains auteurs (Zobell et Feltham, 1938; Fredeen, 1964; Calow, 1974; Lopez et Levinton, 1978) semblent admettre que les bactéries présentes dans les débris organiques constituent une biomasse suffisante pour couvrir les besoins alimentaires des animaux; tandis que d'autres (Fenchel, 1972; Baker et Bradnam, 1976; Wotton, 1980; Newell et Field, 1983) rejettent cette éventualité en faisant remarquer que les bactéries sont trop peu nombreuses dans les bols alimentaires pour représenter une source trophique appréciable. Les bactéries ingérées représenteraient moins de $4 \%$ des besoins alimentaires des larves de diptères Simuliidae (Martin et Kukor, 1984) et moins de $15 \%$ du carbone rejeté par la respiration d'un crustacé isopode d'eau douce (Findlay et al, 1984). Pour Findlay et Tenore (1982), les bactéries constitueraient cependant la principale source d'azote utilisée par les invertébrés dans un milieu où cet élément est un facteur limitant.

Dans tous ces travaux, le rôle éventuel des protozoaires n'est jamais pris en compte. Or, nous avons indiqué précédemment que les protozoaires étaient de bons utilisateurs de la biomasse bactérienne avec des rendements élevés (40-50\%). De plus, les stades actifs (trophozoïtes) qui sont lysés au cours du transit digestif chez les lombriciens (Piearce et Phillips, 1980; Rouelle, 1983) doivent l'être également chez d'autres invertébrés. II ne semble pas cependant que les protozoaires seuls puissent représenter une part importante de l'alimentation des invertébrés du fait de leur faible densité de population. Comme dans le cas des bactéries, il convient de nuancer cette appréciation en considérant le rôle éventuel du choix alimentaire : ainsi le comportement de certains invertébrés qui raclent les surfaces (Lopez et Levinton, 1978) pourrait permettre l'ingestion d'une forte densité de protozoaires sessiles (ciliés, flagellés) ou rampants (amibes).

La biomasse fongique ingérée avec les débris organiques ne couvrirait, elle aussi, qu'une faible partie des besoins alimentaires des invertébrés saprophages, $8 \%$ d'après Martin et Kukor (1984) chez les larves de tipule (diptères, Tipulidae).

Quelle que puisse être leur importance dans l'alimentation des invertébrés, les prélèvements opérés aux dépens de la microflore bactérienne devraient avoir un effet insignifiant sur l'activité microbienne globale du milieu en raison de la faible proportion de la microflore totale qui transite par les tubes digestifs des animaux (Gorbenko et al, 1986). 
Les invertébrés saprophages pourraient enfin se nourrir de substances facilement assimilables provenant de la digestion extracellulaire de la microflore vis-à-vis de laquelle ils se comporteraient alors en parasites. Chez les lombriciens, l'activité métabolique de la microflore en transit dans le tube digestif serait stimulée par la production de mucus; ce dernier agirait par "priming effect" et provoquerait une dégradation microbienne accrue des matières organiques du bol alimentaire, avec des chutes de concentration oscillant entre 9 et $19 \%$ (Lavelle, 1988). Ce phénomène devrait être limité par la dépense énergétique correspondant à la synthèse du mucus. De plus, il convient de préciser que la production de mucus, générale chez les animaux, est interprétée chez I'homme d'une manière très différente (Leclerc et Mossel, 1989) : elle est plus considérée comme un élément de protection de l'organisme que comme un facteur de prolifération bactérienne.

En conclusion, les invertébrés saprophages qui ne peuvent assurer leur subsistance directement à partir des polymères structuraux, se nourriraient de protozoaires, de mycélium, de produits de la digestion extracellulaire de la microflore et éventuellement de bactéries. Cependant, la consommation de ces dernières ne paraît pas suffisante pour agir de façon notable sur les populations bactériennes qui sont les principales concernées par le phénomène de stimulation.

\section{Cas particulier des relations protozoaires - bactéries et mise en évidence d'une médiation chimique}

Dans l'introduction de cet article, nous avons supposé que la stimulation microbienne pourrait être due à la régulation du niveau de population par la prédation. Cette hypothèse devrait être vérifiée facilement dans le cas des protozoaires qui consomment activement les bactéries et qui se prêtent très bien au contrôle rigoureux des conditions expérimentales. Curieusement, les travaux récents consacrés à l'étude des relations protozoaires-bactéries traitent beaucoup plus souvent du problème de dynamique de population que de celui des réactions métaboliques des bactéries.

\section{Prédation et dynamique de population}

L'intérêt particulier porté à cet aspect se justifie par la recherche d'un modèle mathématique permettant de prévoir l'évolution des populations en présence. Cela se justifie à la fois sur le plan fondamental et sur le plan appliqué dans la perspective du traitement biologique des eaux usées ou de l'inoculation des sols avec des bactéries utiles, génétiquement modifiées ou non.

Dans ce dernier cas, les travaux réalisés par Alexander et ses collaborateurs depuis 1975 (voir en particulier Acea et al, 1988) sont particulièrement intéressants : les principales conclusions sont les suivantes. Les protozoaires sont responsables de la diminution du nombre de bactéries lorsqu'elles sont introduites massivement ( $10^{9}-10^{10}$ bactéries/g de sol) dans un sol. Si les bactéries n'ont pas la possibilité de se multiplier, elles peuvent être éliminées (bactéries pathogènes des homéothermes, pouvoir épurateur du sol); dans le cas contraire, la densité de population bactérienne se stabilise à un niveau relativement élevé, de l'ordre de $10^{5}$ bactéries/g de sol (Xanthomonas) ou de $10^{7}$ bactéries/g de sol (Rhizobium). L'état d'équilibre atteint alors est non pas statique mais dynamique, prédateurs et proies conservant un faible taux de multiplication; les pertes par prédation sont compensées par la croissance résiduelle des bactéries. Différents modèles ont été proposés pour traduire ces évolutions de populations (Steinberg, 1987).

De très nombreux travaux ont été réalisés en fermenteur (Sambanis et Fredrickson, 1988) mais toujours en portant plus d'attention aux effectifs qu'aux modifications du métabolisme bactérien.

En conclusion, puisque les protozoaires jouent un rôle important dans la régulation des populations bactériennes, ils constituent un matériel de choix pour savoir si cette régulation est effectivement à l'origine de la stimulation.

\section{Prédation et activité bactérienne}

\section{Travaux anciens et conception classique}

Les travaux sur les modifications du métabolisme bactérien en présence de protozoaires sont peu nombreux et datent presque tous de la première moitié du XXe siècle (Pussard, 1971 ou Coûteaux et al, 1988). La conclusion commune était qu'in vitro l'activité bactérienne est significativement accrue en présence de protozoaires bactériophages. Elle pouvait paraître paradoxale puisqu'en présence de prédateurs, la densité des populations bactériennes est généralement plus faible. Par ailleurs, elle n'est pas conforme à la notion générale de prédation 
qui chez les métazoaires s'accompagne plutôt d'une réduction d'activité de la population de proies. C'est pourquoi ces travaux anciens n'ont guère été pris en considération et cela d'autant que les interprétations des résultats étaient critiquables. En effet, les activités mesurées étaient souvent communes aux bactéries et aux protozoaires (émission de $\mathrm{CO}_{2}$; rejet de $\mathrm{N}-\mathrm{NH}_{4}$ ), d'où la tendance à attribuer aux protozoaires l'excès d'activité observée en culture mixte par rapport à une culture bactérienne pure servant de témoin. Quelques auteurs ont pourtant étudié une fonction spécifiquement bactérienne, la fixation d'azote atmosphérique par Azotobacter. Malheureusement, leurs travaux ont manqué de rigueur (Hervey et Greaves, 1941) et les auteurs ultérieurs (Darbyshire, 1972) n'ont pas retenu leurs conclusions. Récemment, Griffiths (1989) a signalé la stimulation de la nitrification en présence de protozoaires bactériophages qui ne peuvent être suspectés de participer à cette fonction.

Pour expliquer le phénomène de stimulation, les mécanismes les plus divers ont été proposés sans véritable vérification : maintien d'une faible densité de population bactérienne et/ou d'une forte proportion de bactéries jeunes, modification du $\mathrm{pH}$ du milieu, rejet de substances stimulantes par les protozoaires. Dans la littérature, il existe toutefois des exceptions à la règle de stimulation : ainsi Huang et al (1981) ont observé une diminution de la dégradation du paraaminobenzoate par la bactérie Alcaligenes en présence de Tetrahymena.

Indépendamment de ces travaux, une équipe pluridisciplinaire de Fort-Collins (Coleman et al, 1984) et d'Uppsala (Clarholm, 1985) a entrepris l'étude des interactions entre nématodes, protozoaires et bactéries en microcosmes gnotoxéniques. L'objectif était d'étudier l'effet de ces interactions sur la minéralisation de la matière organique en s'inspirant de travaux réalisés en milieu aquatique (Jost et al, 1973; Fenchel et Jorgensen, 1977). L'intensification de la minéralisation de l'azote et du phosphore observée en présence de protozoaires est attribuée à l'excrétion de $\mathrm{N}-\mathrm{NH}_{4}$ et de phosphates par ces derniers. Les auteurs admettent implicitement que l'activité métabolique des bactéries n'est en rien modifiée en présence de protozoaires; seule interviendrait la consommation de corps bactériens. Ce postulat de l'invariabilité du comportement bactérien a été contesté par Coûteaux et al (1988) qui ont attribué l'excès de $\mathrm{CO}_{2}$ produit en culture mixte non pas à la respiration des proto- zoaires, considérée comme négligeable, mais à une croissance bactérienne supplémentaire, masquée par les prélèvements dus à la prédation et appelée pour cette raison croissance "cryptique». Ainsi la production bactérienne serait effectivement plus grande en présence de prédateurs (Pussard et Rouelle, 1986). La croissance cryptique serait possible du fait de la formation de substrats nutritifs supplémentaires provenant de la digestion incomplète de la biomasse bactérienne consommée par les prédateurs (substances autochtones de Sambanis et al, 1987). Ainsi, la conception classique considère que la stimulation est le résultat de l'excrétion - défécation de substrats par les protozoaires et qu'elle est liée à une augmentation de la production bactérienne. Cette interprétation est conforme à celle admise actuellement pour expliquer le rôle des invertébrés dans le sol. Kuikman (1990) admet toutefois qu'il existe aussi un effet de stimulation du métabolisme des bactéries survivantes indépendamment de l'effet lié au recyclage partiel de la biomasse consommée sans toutefois proposer d'hypothèse sur le mécanisme en jeu.

\section{Démonstration de la médiation chimique}

Dans un récent travail de thèse, Levrat (1990b) a étudié le mécanisme de la stimulation sur un modèle biologique favorable constitué par une amibe bactériophage Acanthamoeba castellanii et une bactérie prototrophe (aucun besoin en facteur de croissance) fluorescente du genre Pseudomonas. L'activité de la population bactérienne a été suivie grâce à la production de pigment fluorescent (pyoverdine à propriétés de sidérophore) spécifique des bactéries et facile à doser par spectrophotométrie. Dans un milieu de culture (milieu B de King dilué au 1/10) permettant la croissance simultanée des 2 microorganismes, la concentration de pigment est environ 2 fois plus élevée en culture mixte qu'en culture pure (Levrat et al, 1989). Ce même résultat peut être intégralement reproduit en culture pure de bactéries par addition de $2-5 \%$ de filtrat acellulaire de culture mixte ou de culture axénique d'amibes (Levrat, 1990a). Si la proportion de filtrat dépasse $2 \%$, la production de pigment n'est pas augmentée pour autant. Autre fait capital, dans ces cultures pures additionnées de filtrats de culture mixte, donc en absence de prédation, le niveau de population bactérienne n'est pas différent de celui du témoin. Enfin, puisque la production de pyoverdine dépend, entre autres facteurs, de la disponibilité en fer ferrique du milieu 
de culture (Meyer, 1977), vérification a été faite qu'aucune diminution de concentration de cet élément ne pouvait expliquer les augmentations de la production de pigment observées.

Dans l'ensemble, ces résultats montrent que la stimulation des bactéries ne résulte pas de l'utilisation de substrats «autochtones» et qu'elle n'est pas en rapport avec une augmentation de la production de biomasse bactérienne. II était d'ailleurs prévisible qu'il ne pouvait en être autrement : comment supposer en effet que les amibes dont la respiration est négligeable par rapport à celle des bactéries (Coûteaux et al, 1988) puissent être capables de libérer assez de substrats nutritifs supplémentaires pour soutenir un doublement de l'activité bactérienne ? Les résultats obtenus par Levrat permettent aussi d'éliminer sans ambiguitté l'hypothèse d'une stimulation métabolique dépendante d'une variation de la densité de population bactérienne. En définitive, c'est donc l'émission de substances stimulantes par les protozoaires qui permet seule d'expliquer les phénomènes observés. Ces substances agiraient à faible concentration en modifiant l'activité métabolique de chaque bactérie. Leur nature chimique et leur mode d'action sont à l'étude.

Par ailleurs, Levrat (1990a) a montré que l'addition de filtrat de culture mixte stimule non seulement la production de pigment mais aussi l'activité respiratoire et la libération de $\mathrm{N}-\mathrm{NH}_{4}$ dans la culture pure de Pseudomonas. Ceci confirme le rôle attribué aux protozoaires de la rhizosphère dans la nutrition azotée des plantes tout en mettant en échec l'interprétation proposée par Woods et al (1982) et par Clarholm (1985) qui attribuaient l'essentiel de la production de $\mathrm{N}-\mathrm{NH}_{4}$ à l'excrétion azotée des protozoaires. Ainsi les substances émises par les amibes stimulent chez les bactéries non seulement la production d'un métabolite secondaire, le pigment, mais également le rejet de produits du catabolisme $\left(\mathrm{CO}_{2}, \mathrm{NH}_{4}{ }^{+}\right)$et cela sans accroître de façon sensible la synthèse de constituants cellulaires (absence d'accroissement de biomasse). On peut conclure avec Fenchel (1977) «thus it seems that grazing of the bacteria change their physiology".

Ces conclusions permettent de concevoir d'une façon originale les relations entre protozoaires et bactéries puisque la prédation au sens strict n'en constituerait plus l'aspect essentiel. Certes les protozoaires sont des prédateurs de bactéries et ils se comportent bien en prédateurs "prudents» au sens de Slobodkin (1968) puisqu'ils ne s'attaquent qu'aux populations dont la densité dépasse le seuil de $10^{5}-10^{7}$ bactéries $/ g$ de sol. À l'évidence, ce comportement contribue au maintien de la diversité des peuplements microbiens.

Par contre, l'essentiel des relations entre protozoaires et bactéries reposerait sur le phénomène de stimulation du métabolisme bactérien. Cette stimulation semble être tout à fait générale puisque, comme nous l'avons indiqué ci-dessus, de très nombreux auteurs sont parvenus à cette conclusion en travaillant dans les conditions les plus diverses. En présence de protozoaires, qu'il y ait ou non prédation aux dépens de la population bactérienne, l'aptitude à la compétition de cette dernière serait accrue : un exemple de ce phénomène a été signalé par Levrat et al (1987) à propos du renforcement, par les amibes, de l'antagonisme d'un Pseudomonas vis-à-vis de Fusarium. Ce faisant, les protozoaires favorisent la survie des populations bactériennes dont ils se nourrissent.

Ainsi, grâce à un principe actif diffusant dans le milieu, les protozoaires modifient le métabolisme bactérien. De même, certaines espèces de Ciliés émettent des substances qui provoquent la transformation morphologique d'autres Ciliés en rendant tantôt les prédateurs capables de capturer des proies plus volumineuses (Buhse, 1967), tantôt les proies potentielles plus difficiles à ingérer par les espèces prédatrices (Kuhlmann et Heckmann, 1985). Ces quelques exemples permettent de soupçonner l'importance des médiateurs chimiques en écologie microbienne et d'illustrer la complexité des relations prédateurs-proies chez les microorganismes.

Dans le cas étudié ici, la prédation n'a pas les conséquences négatives habituelles : elle relève plutôt de la coopération et correspond à une forme de prédation très évoluée en rapport avec la particulière ancienneté de la coexistence des bactéries et des protozoaires. On peut supposer qu'au cours de cette très longue cohabitation l'évolution ait sélectionné les populations bactériennes les plus aptes à profiter de certaines substances rejetées par les protozoaires.

\section{Hypothèse de l'émission de substances stimulantes par les invertébrés}

Il est logique d'étendre les considérations précédentes au cas des invertébrés : pourquoi l'évolution n'aurait-elle pas aussi sélectionné les popu- 
lations bactériennes les plus aptes à tirer avantage des substances rejetées par les invertébrés?

Une telle démarche permettrait d'expliquer de façon satisfaisante le paradoxe fonctionnel des relations faune-microflore sans enfreindre les principes élémentaires de la biologie : l'émission de substances stimulantes dont l'action ne serait pas en rapport avec leur concentration, contrairement aux substrats nutritifs, serait compatible avec la faiblesse relative du budget énergétique d'une population animale. II deviendrait également possible d'expliquer les cas de stimulation d'activité bactérienne correspondant plutôt à une diminution de la densité de population (Barsdate et al, 1974; Anderson et al, 1978; Baath et al, 1981; Anderson et al, 1983). L'effet de substances stimulantes serait localisé au milieu intestinal et/ou au voisinage immédiat de l'animal. II ne dépendrait pas nécessairement de la bactériophagie mais de toute évidence il contribuerait à améliorer la nutrition de l'invertébré saprophage dans la mesure où les métabolites bactériens produits en plus grande quantité représentent une part importante de son alimentation.

Cette hypothèse qui propose un mécanisme de stimulation de la microflore identique pour toute la série animale n'est pas en opposition avec l'homogénéité de la réponse de la microflore lorsqu'elle est en présence des divers groupes zoologiques aux comportements si variés : ainsi la nitrification est accrue en présence d'animaux aussi différents que les protozoaires, les diplopodes, les collemboles et les lombriciens (Syers et al, 1979; Anderson et al, 1983; Griffiths, 1989). De plus, l'émission de substances allélochimiques expliquerait certaines observations mieux que ne peut le faire la théorie du rejet de substrats nutritifs. C'est ainsi que Rouelle et al (1985) oni constaté que l'introduction du lombricien Eisenia fetida andrei dans un compost urbain mûr ne provoque qu'une très faible reprise d'activité microbienne (minéralisation de l'azote; multiplication des protozoaires); par contre, l'apport de phosphore permet une nette relance de cette activité qui est alors fortement accrue si les lombriciens sont présents. Ces résultats montrent que l'émission de substrats nutritifs (sécrétions, excrétions) par les lombriciens a globalement peu d'effet sur la microflore d'un milieu dont l'activité est très ralentie par manque de nutriment. Par contre, après apport du nutriment limitant, les lombriciens s'avèrent capables d'accroître significativement l'activité microbienne, ce qui est compatible avec l'hypothèse de l'émission par eux de substances stimulantes.

Le mécanisme envisagé ici pour expliquer l'effet de stimulation de la microflore par la faune présente l'avantage d'avoir été déjà démontré dans le cas des protozoaires, ce qui n'est pas le cas des autres théories admises actuellement.

II est intéressant de souligner le fait que, si l'hypothèse proposée ici était vérifiée, elle constituerait un argument de plus contre le postulat de l'invariabilité du comportement de la microflore auquel on se réfère si souvent et que nous avons dénoncé à propos des travaux sur les protozoaires. Dans le cas des invertébrés aussi, l'observation de ce postulat conduit vraisemblablement à des conclusions erronées. Ainsi, certains auteurs (Cortez et al, 1989) attribuent à une action directe des vers de terre sur la matière organique une augmentation d'activité qui relèverait plutôt de la stimulation de la microflore en présence de ces animaux : l'interprétation de ces auteurs a l'inconvénient de perpétuer l'idée que les vers de terre sont des décomposeurs primaires au moins aussi actifs que la microflore, bien que cela soit contraire aux caractéristiques du modèle animal et n'ait jamais été prouvé.

Pour vérifier l'hypothèse proposée et mettre en évidence des substances stimulantes d'origine animale, il sera indispensable de disposer au préalable de techniques efficaces de désinfection des invertébrés. II est en effet nécessaire d'éliminer toute interférence avec les microorganismes d'accompagnement et particulièrement celle avec les protozoaires. Or, cette opération est d'autant plus délicate que les invertébrés dépendent, pour leur nutrition, de la microflore associée.

La stimulation de la microflore par des substances d'origine animale pourrait expliquer des phénomènes très divers dont nous donnons les 2 exemples suivants.

En premier lieu, une activité thiaminolytique (facteur anti-vitamine B1) a été détectée chez le lombricien Eisenia fetida andrei (Rouelle et al, 1987; Rouelle et Lhuissier, 1988). Elle proviendrait de la stimulation, au cours du transit intestinal, de bactéries thiaminolytiques présentes dans les déchets organiques en fermentation où elles sont normalement peu actives. II pourrait s'agir d'une adaptation conférant à l'espèce lombricienne une aptitude accrue à coloniser des $\mathrm{mi}$ lieux très fermentescibles en réduisant les nuisances liées à l'activité de la microflore opportuniste à faible pouvoir de synthèse. En se- 
cond lieu, la dégradation de minéraux argileux au cours du transit intestinal de certains invertébrés (Leprun et Roy-Noël, 1976; Boyer, 1982; Rouelle et Randriamamonjizaka, 1983) pourrait être due, au moins en partie, à la stimulation d'une microflore spécialisée (bactéries "silicatées" de Tesic, 1972).

Dans tout ce qui précède, nous n'avons envisagé que les relations de la faune avec la microflore bactérienne. Nous ne disposons d'aucun indice qui permette de supposer que des relations de même nature existent avec les champignons. Par contre, la faune devrait plutôt avoir un effet dépressif sur l'activité fongique par l'intermédiaire de la stimulation de l'antagonisme bactérien : ce pourrait même être là une explication d'un phénomène assez fréquent (Hanlon et Anderson, 1979 et 1980; Satchell, 1983), à savoir l'effet de substitution de la microflore fongique par la microflore bactérienne en présence d'animaux.

L'importance d'une meilleure connaissance des interactions entre organismes différents apparaît clairement. Ne considérer que des espèces isolées en culture ou en élevage comporte le risque de ne révéler qu'une version très limitée des phénomènes qui se produisent en situation naturelle, dans un environnement biologique complexe. Alors que l'usage du microsome se répand, il convient de critiquer tout autant les expérimentations pratiquées avec des invertébrés comme si la microflore n'existait pas que celles sur la microflore en absence de toute influence animale.

\section{CONCLUSIONS}

Le rôle des animaux, et plus spécialement celui des protozoaires, dans les processus de biodégradation consisterait en une intensification importante du métabolisme de la microflore bactérienne. Cet effet pourrait se manifester non seulement dans le milieu ambiant (sol, litière, sédiment, eau douce, eau de mer) mais également dans le tube digestif des animaux où coexistent souvent bactéries et protozoaires symbiotiques. Dans ce cas l'activité microbienne pourrait être stimulée à la fois par les protozoaires et par les sécrétions de l'hôte lui-même. La notion proposée ici aurait donc un domaine d'application très vaste, dépassant largement le cadre de la biologie du sol. En raison de leur ubiquité et de leurs caractéristiques biologiques, les protozoaires devraient jouer un rôle prépondérant dans la stimulation des bactéries.
La mise en évidence de la stimulation des bactéries par les protozoaires et l'hypothèse d'une action identique exercée par les métazoaires permettent d'intégrer de façon logique l'activité de l'ensemble des animaux dans les mécanismes microbiens de la nutrition des plantes et de la fertilité des sols.

La dépendance absolue de la faune saprophage vis-à-vis de la microflore (besoins en vitamines et en enzymes) est assez bien admise. $\dot{A}$ l'inverse, la stimulation du métabolisme bactérien en présence d'animaux constitue un domaine de recherche nouveau, totalement négligé jusqu'ici.

Une meilleure connaissance des interactions biochimiques entre faune et microflore serait fructueuse non seulement sur le plan fondamental de l'écologie mais également sur le plan appliqué en lutte microbiologique (utilisation de l'antagonisme bactérien pour lutter contre les fusarioses vasculaires) et même en biotechnologie dans la mesure où il serait permis d'espérer obtenir de meilleurs rendements en métabolites bactériens dans nos fermenteurs.

In the era of biotechnology we can probably expect the protozoa to teach us something about exploiting microbes.

Cette prophétie de Finlay et Fenchel (1989) pourrait s'appliquer à tous les animaux et entrer dans la réalité beaucoup plus rapidement que ne le prévoyaient ces auteurs.

\section{REMERCIEMENTS}

L'auteur a très largement bénéficié de la réflexion de $\checkmark$ Rouelle sur de nombreux problèmes évoqués dans $c e$ texte. Il lui en est très reconnaissant.

\section{RÉFÉRENCES}

Acea MJ, Moore CR, Alexander M (1988) Survival and growth of bacteria introduced into soil. Soil Biol Biochem 20, 509-515

Alabouvette C, Lemaître I, Pussard M (1981) Densité de population de l'amibe mycophage Thecamoeba granifera s sp minor (Amoebida, Protozoa) Mesure et variations expérimentales dans le sol. Rev Écol Biol Sol 18, 179-192

Anderson RV, Elliott ET, Macclellan JF, Coleman DC, Cole CV, Hunt HW (1978) Trophic interactions in soils as they affect energy and nutrient dynamics. III. Biotic interactions of bacteria, amoebae and nematodes. Microb Eco/4, 361-371 
Anderson JM, Ineson P, Huish SA (1983) Nitrogen and cation mobilization by soil fauna feeding on leaf litter and soil organic matter from deciduous woodlands. Soil Biol Biochem 15, 463-467

Austin DA, Baker JH (1988) Fate of bacteria ingested by larvae of the freshwater may fly, Ephemera danica. Microb Ecol 15, 323-332

Baath E, Lohm U, Lundgren B, Sohlenius B (1981) Impact of microbial-feeding animals on total soil activity and nitrogen dynamics: a soil microcosm experiment. Oikos 37, 257-264

Baker JH, Bradnam LA (1976) The role of bacteria in the nutrition of aquatic invertebrates. Oecologia 24, 95-104

Bärlocher F, Kendrick B (1975) Leaf-conditioning by microorganisms. Oecologia 20, 359-362

Barsdate RJ, Prentki RT, Fenchel T (1974) Phosphorus cycle of model ecosystems: significance for decomposer food chains and effect of bacteria grazers. Oikos 25, 239-251

Bassalik K (1913) Uber Silikatzersetzung durch Bodenbakterien. I. Uber die Tätigkeit der Regenwürmer in Beziehung zu den Bodenbakterien. $Z f$ Gärungsphysiol 2, 1-32

Bignell DE (1989) Relative assimilations of ${ }^{14} \mathrm{C}$ labelled microbial tissues and ${ }^{14} \mathrm{C}$-plant fibre ingested with leaf litter by the millipeda Glomeris marginata under experimental conditions. Soil Biol Biochem 21, 819-827

Boyer P (1982) Quelques aspects de l'action des termites du sol sur les argiles. Clay Miner 17, 453-462

Brüsewitz G (1959) Untersuchungen über den Einfluss des Regenwurms auf Zahl, Art und Leistungen von Mikroorganismen im Boden. Arch Mikrobiol 33, 52-82

Buhse HE Jr (1967) Microstome-macrotome transformation in Tetrahymena vorax strain V2 type $S$ induced by a transforming principle, stomatin. $J$ Protozool 14, 608-613

Calow P (1974) Evidence for bacterial feeding in Planorbis contortus Linn (Gastropoda: Pulmonata). Linn Proc Malac Soc (Londres) 4, 145-156

Cameron GR (1932) Inflammation in earthworms. $J$ Pathol Bacterio/ 35, 933-972

Childs M, Bowman CE (1981) Lysozyme activity in six species of economically important astigmated mites. Comp Biochem Physio/ B, 70, 615-617

Chu TL, Szabo IM, Szabo I (1987) Nocardioform gut actinomycetes of Glomeris hexasticha Brandt (Diplopoda). Biol Fertil Soils 3, 113-116

Clarholm M (1985) Interactions of bacteria, protozoa and plants leading to mineralization of soil nitrogen. Soil Biol Biochem 17, 181-187

Coleman DC, Anderson RV, Cole CV, Macclellan JF, Woods LE, Trofymow JA, Elliot ET (1984) Roles of protozoa and nematodes in nutrient cycling. $I n$ : $M i$ crobial-Plant interactions. ASA Spec Pub $n^{\circ} 47,17$ 28
Cooke A, Luxton M (1980) Effect of microbes on food selection by Lumbricus terrestris. Rev Ecol Biol Sol $17,365-370$

Cortez J, Hameed R, Bouché MB (1989) C and N transfer in soil with or without earthworms fed with ${ }^{14} \mathrm{C}$ - and ${ }^{15} \mathrm{~N}$-labelled wheat straw. Soil Biol Biochem 21, 491-497

Coûteaux MM, Faurie G, Palka L, Steinberg C (1988) La relation prédateur-proie (protozoaires-bactéries) dans les sols : rôle dans la régulation des populations et conséquences sur les cycles du carbone et de l'azote. Rev Ecol Biol Sol 25, 1-31

Darbyshire JF (1972) Nitrogen fixation by Azotobacter chroococcum in the presence of Colpoda steini-l. The influence of temperature. Soil Biol Biochem 4, 359-369

Dawson RC (1948) Earthworm microbiology and the formation of water-stable aggregates. Soil Sci Soc Am Proc 12, 512-516

Day GM (1950) Influence of earthworms on soil microorganisms. Soil Sci69, 175-184

Ducluzeau R, Raibaud P (1979) Ecologie microbienne du tube digestif. Actualités Scientifiques et Agronomiques de l'INRA, Masson, Paris, $94 \mathrm{p}$

Duhlinska DD (1979) Cultivation of some Lumbricidae uninfected with monocystid gregarines. Acta Zool Bulg 12, 78-80

Elliott ET, Anderson RV, Coleman DC, Cole CV (1980) Habitable pore space and microbial trophic interactions. Oikos 35, 327-335

Fenchel TM (1972) Aspects of decomposer foodchains in marine benthos. Verh Dtsch Zool Ges 65 Jahresversamml 14, 14-22

Fenchel TM (1977) The significance of bactivorous protozoa in the microbial community of detrital particles. In: Aquatic microbial communities (Cairns J, ed) Garland Publ NY et Londres, 529-543

Fenchel TM, Jorgensen BB (1977) Detritus food chains of aquatic ecosystems: the role of bacteria. Adv Microb Ecol 1, 1-58

Findlay S, Tenore K (1982) Nitrogen sources for a detritivore: detritus substrate versus associated microbes. Science 218, 371-373

Findlay S, Meyer J, Smith P (1984) Significance of bacterial biomass in the nutrition of a freshwater isopod (Lirceus sp). Oecologia 63, 38-42

Finlay B, Fenchel TM (1989) Everlasting picnic for protozoa. New Sci, 1 er juillet, 66-69

Flack FM, Hartenstein R (1984) Growth of the earthworm Eisenia foetida on microorganisms and cellulose. Soil Biol Biochem 16, 491-495

Foissner W, Didier P (1983) Nahrungsaufnahme, Lebenszyklus und Morphogenese von Pseudoplatyophrya nana (Kahl, 1926) (Ciliophora, Colpodida). Protistologica 19, 103-109

Fredeen FJH (1964) Bacteria as food for blackfly larvae (Diptera: Simuliidae) in laboratory cultures and in natural streams. Can J Zool 42, 527-548 
Gorbenko AY, Panikov NS, Zvyagintsen DV (1986) The effect of invertebrates on the growth of soil $\mathrm{mi}$ croorganisms. Mikrobiologia 55, 515-521

Griffiths BS (1989) Enhanced nitrification in the presence of bacteriophagous protozoa. Soil Biol Biochem 21, 1045-1051

Hanlon RDG, Anderson JM (1979) The effects of collembola grazing on microbial activity in decomposing leaf litter. Oecologia 38, 93-99

Hanlon RDG, Anderson JM (1980) The influence of macroarthropod feeding activities on microflora in decomposing oak leaves. Soil Biol Biochem 12, 255-261

Hargrave BT (1970) The utilization of benthic microflora by Hyallela azteca (Amphipoda). J Anim Ecol 39, 427-437

Harper RM, Fry JC, Learner MA (1981a) Digestion of bacteria by Nais variabilis (Oligochaeta) as established by autoradiography. Oikos 36, 211-218

Harper RM, Fry JC, Learner MA (1981b) A bacteriological investigation to elucidate the feeding biology of Nais variabilis (Oligochaeta: Naididae). Freshw Biol 11, 227-236

Hassett DJ, Bisesi MS, Hartenstein R (1988) Earthworm peroxidase distribution, microbicidal action and molecular weight. Soil Biol Biochem 20, 887 890

Hervey RJ, Greaves JE (1941) Nitrogen fixation by Azotobacter chroococum in the presence of soil protozoa. Soil Sci 51, 85-100

Huang TC, Chang MC, Alexander M (1981) Effect of protoza on bacterial degradation of an aromatic compound. App/ Environ Microbiol 41, 229-232

Jost JL, Drake JF, Tsuchiya JM, Fredrickson AG (1973) Microbial food chains and food webs. J Theor Biol 41, 461-484

Kaushik NK, Hynes HBN (1971) The fate of the dead leaves that fall into streams. Arch Hydrobiol 68, 465-515

Kristufek V, Pizl V, Szabo IM (1989) Composition and properties of the intestinal streptomycete microflora of earthworms (Lumbricidae). In: Collog Int Microbiol Poecilothermes, Paris, 27

Kuhlmann HW, Heckmann K (1985) Interspecific morphogens regulating prey-predator relationships in protozoa. Science 227, 1347-1349

Kuikman PJ (1990) Mineralization of nitrogen by protozoan activity in soil. Thèse, Wageningen, $105 p$

Lavelle $P$ (1988) Earthworm activities and the soil system. Biol Fertil Soil 6, 237-251

Leclerc H, Mossel DAA (1989) Microbiologie. Le tube digestif, l'eau et les aliments. Doin, Paris, $529 \mathrm{p}$

Leprun JC, Roy-Noël J (1976) Minéralogie des argiles et répartition des nids épigés de deux espèces du genre Macrotermes au Sénégal occidental (Presqu'île du Cap Vert). Insectes Soc, Paris, 23, 535547

Levrat $P$ (1990a) Stimulation of the metabolism of a strain of fluorescent Pseudomonas by a bacterio- phagous amoeba (Protozoa, Amoebida). In: Microbiology in poecilotherms (Lesel R, ed) Elsevier Science Publishers, BV Amsterdam

Levrat P (1990b) Contribution à l'étude des interactions entre protozoaires et microflore du sol : effet d'une amibe bactériophage Acanthamoeba castellanii sur le métabolisme du Pseudomonas fluorescens. Thèse, Lyon, $115 p$

Levrat P, Alabouvette C, Pussard M (1987) Rôle des protozoaires dans les systèmes interactifs : cas des relations bactéries-champignons. Rev Ecol Biol Sol 24, 503-514

Levrat $P$, Pussard M, Alabouvette C (1989) Action d'Acanthamoeba castellanii (Protozoa: Amoebida) sur la production de sidérophores par la bactérie Pseudomonas putida. C $R$ Séances Acad SCi Paris, 308, ser III, 161-164

Lopez GR, Levinton JS (1978) The availability of microorganisms attached to sediment particles, as food for Hydrobia ventrosa Montagu (Gastropoda: Prosobranchia). Oecologia 32, 263-275

MacHenery JG, Brikbeck TH, Allen JA (1979) The occurence of lysozyme in marine bivalves. Comp Biochem Physiol B 63, 25-28

Mackay RJ, Kalff J (1973) Ecology of two related species of caddis fly larvae in the organic substrates of a woodland stream. Ecology 54, 499-511

Malke H (1965) Uber das Vorkommen von Lyzozym in Insekten. Z Allg Mikrobiol 5, 42-47

Martin MM, Kukor JJ (1984) Role of mycophagy and bacteriophagy in invertebrate nutrition. In: Current perspectives in microbial ecology (Klug MJ, Reddy CA, eds) Am Soc Microbiol, Washington DC

Meyer JM (1977) Pigment fluorescent et métabolisme du fer chez Pseudomonas fluorescens. Thèse d'état, Strasbourg, $207 \mathrm{p}$

Miles HB (1963) Soil protozoa and earthworm nutrition. Soil Sci 95, 407-409

Newell RC, Field JG (1983) The contribution of bacteria and detritus to carbon and nitrogen flow in a benthic community. Mar Biol Lett 4, 23-36

Old KM (1977) Giant soil amoebae cause perforation of Cochliobolus sativus. Trans Br Mycol Soc 68, 277-320

Old KM, Darbyshire JF (1980) Arachnula impatiens Cienk a mycophagous giant amoeba from soil. Protistologica 16, 277-287

Parle JN (1963) Micro-organisms in the intestines of earthworms. J Gen Microbio/ 31, 1-11

Piearce TG, Phillips MJ (1980) The fate of ciliates in the earthworm gut: an in vitro study. Microb Ecol 5, 313-319

Pussard M (1971) Les protozoaires du sol. In: La vie dans les sols; Aspects nouveaux; Études expérimentales (Pesson P, ed) Gauthier-Villars, Paris, 147-185

Pussard M (1991) Faune du sol et microflore. I. Modèle animal et paradoxe fonctionnel. Agronomie $11,315-324$ 
Pussard M, Rouelle $J$ (1986) Prédation de la microflore. Effet des protozoaires sur la dynamique de population bactérienne. Protistologica 22, 105-110

Pussard M, Alabouvette C, Pons R (1979) Étude préliminaire d'une amibe mycophage, Thecamoeba granifera ssp minor (Thecamoebidae, Amoebida). Protistologica 15, 139-149

Reyes VG, Tiedje JM (1976) Ecology of the gut microbiota of Tracheoniscus rathkei. Pedobiologia 16, 67-74

Rouelle J (1983) Introduction of amoebae and Rhizobium japonicum into the gut of Eisenia fetida (Sav) and Lumbricus terrestris L. In: Earthworm ecology from Darwin to vermiculture (Satchell JE, ed) Chapman and Hall, Londres, 375-381

Rouelle J, Randriamamonjizaka JL (1983) Recherche sur les fèces des vers de terre. In: New trends in soil biology (Lebrun P, André HM, De Medts A, Gregoire-Wibo CG, Wauthy G, eds) Dieu-Brichart, Ottignies Louvain la Neuve, 608-609

Rouelle J, Lhuissier M (1988) Le pouvoir de destruction de la thiamine par le lombricien Eisenia fetida et ses conséquences sur la qualité des composts. C R Acad Agric Fr 74, 103-108

Rouelle J, Pussard M, Randriamamonjizaka JL, Loquet $M$, Vinceslas $M$ (1985) Interactions microbiennes (bactéries, protozoaires), alimentations des vers de terre et minéralisation de la matière organique. Bull Ecol 16, 83-88

Rouelle J, Lhuissier M, Roch P (1987) Experimental evidence for antithiamine activity in certain earthworms. In: On Earthworms (Bonvicini Pagliai AM, Omodeo P, eds) Mucchi ed Bologne, 511-517

Sambanis A, Fredrickson AG (1988) Persistence of bacteria in the presence of viable, non encysting bacteriovorous ciliates. Microb Ecol 16, 197-211

Sambanis A, Pavlou S, Fredrickson AG (1987) Coexistence of bacteria and feeding ciliates. Growth of bacteria on autochthonous substrates as a stabilizing factor for coexistence. Biotechnol Bioeng 29, 714-728

Satchell JE (1983) Earthworm microbiology. In: Earthworm ecology from Darwin to vermiculture (Satchell JE, ed) Chapman and Hall, Londres, 351-364
Seastedt TR (1985) Maximization of primary and secondary productivity by grazers. Ann Nat 126,559 564

Slobodkin LB (1968) How to be a predator? Am Zool 8, 43-51

Sohlenius B (1968) Influence of microorganisms and temperature upon some rhabditid nematodes. $\mathrm{Pe}$ dobiologia 8, 137-145

Steinberg C (1987) Dynamique d'une population bactérienne introduite dans le sol : régulation par les protozaires et modélisation mathématique de la relation de prédation Bradyrhizobium japonicumamibes indigènes. Thèse de doctorat, Lyon, $113 p$

Stephenson W (1942) On the culturing of Rhabditis terrestris n sp. Parasitology 34, 246-252

Swift MJ, Heal OW, Anderson JM (1979) Decomposition in terrestrial ecosystems. Studies in ecology, 5. Blackwell Sci Pub, Oxford, 372 p

Syers JK, Sharpley AN, Keeney DR (1979) Cycling of nitrogen by surface-casting earthworms in a pasture ecosystem. Soil Biol Biochem 1, 181-185

Szabo IM (1989) The indigenous intestinal bacteria of soil arthropods and worms. In: Colloq Int Microbiol Poecilothermes, Paris, 22

Tesic ZP (1972) Utilisations possibles des bactéries silicatées dans l'étude de certains problèmes de la microbiologie du sol. Rev Ecol Biol Sol 9, 589-593

Wavre M, Brinkhurst RO (1971) Interactions between some tubificid oligochaetas and bacteria found in the sediments of Toronto Harbour, Ontario. $J$ Fish Res Board Can 28, 335-341

Woods LE, Cole CV, Elliott ET, Anderson RV, Coleman DC (1982) Nitrogen transformations in soil as affected by bacterial-microfaunal interactions. Soil Biol Biochem 4, 93-98

Wotton RS (1980) Bacteria as food for blackfly larvae (Diptera: Simuliidae) in a lake outlet in Finland. Ann Zool Fenn 17, 127-130

Wright MA (1972) Factors governing ingestion by the earthworm Lumbricus terrestris (L), with special reference to apple leaves. Ann Appl Biol 70, 175-188

Zobell CE, Feltham CB (1938) Bacteria as food for certain marine invertebrates. J Mar Res 1, 312-327 